\title{
Una aproximación a la contabilidad del capital intelectual en el proceso de toma de decisiones: un estudio en el Oriente antioqueño colombiano
}

\author{
Carlos Eduardo Castaño Rios ${ }^{17}$; Paola Andrea \\ Reinoso Sánchez ${ }^{18}$; Jahir Alberto Zapata Gallo ${ }^{19}$; \\ John Jairo del Rio Aguirre ${ }^{20}$ \\ Colombia
}

\section{Resumen}

El capital intelectual es un tema poco conocido por los tomadores de decisiones en el contexto empresarial colombiano. Establecer las implicaciones que tiene para la toma de decisiones gerenciales el reconocimiento del capital intelectual dentro de los informes contables, es lo que se pretende abordar en el presente trabajo, para ello se realizó un grupo de discusión que contó con la presencia de un grupo de individuos de diferentes empresas de la región del oriente antioqueño, con el perfil de tomadores de decisiones. Por medio de sus puntos de vista se logró evidenciar lo novedoso que es el tema para estas personas y del escaso acceso que tienen a información relacionada con el capital intelectual. El reconocimiento de estos recursos de gran valor para la organización a través del sistema de información contable permite incrementar el poder informativo de la contabilidad y dar soluciones puntuales a temas que deben ser tratados a diario en la toma de decisiones empresariales.

\footnotetext{
${ }^{17}$ Profesor Universidad de Antioquia (Colombia). Email: castano@udea.edu.co

${ }^{18}$ Contadora pública Universidad de Antioquia (Colombia). Email: pa-reinoso@hotmail.com

${ }^{19}$ Contador público Universidad de Antioquia (Colombia). Email: jhircoria01@hotmail.com

${ }^{20}$ Contador público Universidad de Antioquia (Colombia). Email: johnjadra@hotmail.com
}

Recibido: 12/02/2015 - Versión final aceptada: 29/04/2015 
Palabras clave: capital intelectual, toma de decisiones, informes de capital intelectual, sistema de información contable, informes contables.

\begin{abstract}
Intelectual capital is a topic which is little known by decision makers in the Colombian business context. On this paper, we would like to establish the implications the acknowledgement of intelectual capital has in management decision making within accounting reports, in order to do this, a discussion group with members from different companies in Eastern Antioquia was formed, with decision making profiles. Through their points of view, it was shown how novel this subject is for these individuals and how scarce it is to access information related to intelectual capital. The acknowledgement of these resources, which are highly valued by the organization through its accounting information system allows the possiblity to increase the informative power of accounting and to give accurate solutions to topics which should be daily addressed in business decision making.
\end{abstract}

Key words: intelectual capital, decision making, intelectual capital reports, accounting information system, accounting reports.

\title{
Introducción
}

Los esquemas de las organizaciones en el siglo XXI, desde sus estructuras operativas y jerárquicas hasta la forma de tomar y adoptar decisiones estratégicas, han cambiado por la nfluencia de fuerzas económicas, políticas, culturales y sociales. Las nuevas dinámicas revolucionan la mirada tradicional de los recursos organizacionales y abre el panorama de la competitividad de las empresas y sus procesos de valoración, esto movido por la nueva tendencia de exaltar el conocimiento, los avances tecnológicos y el flujo de información, como las principales fuentes generadoras de valor.

La nueva era del conocimiento y la información crea la necesidad de que los administradores replanteen su forma de tomar decisiones y basen estas en los recursos de importancia en su organización y la mejor forma de hacerlo es seleccionando información pertinente. Es por esto que la contabilidad, como mayor insumo de información en la empresa, tiene la posibilidad de ampliar su campo de acción y cumplir 
su objetivo de apoyar a la administración en sus labores incluyendo dentro de sus informes los referentes al capital intelectual.

Esta oportunidad potencial le da vida a este trabajo investigativo que partió del objetivo de establecer las implicaciones que tiene para la toma de decisiones gerenciales el reconocimiento del capital intelectual dentro de los informes contables. El trabajo se desarrolla en una línea de contabilidad y gestión con base en una investigación de enfoque cualitativo a partir de la relación entre una nueva información contable no financiera y su influencia en el proceso de toma de decisiones por parte de los encargados de esta labor.

Para llegar al resultado que aquí se presenta se hizo necesario abordar de manera separada los temas de contabilidad del capital intelectual y el de toma de decisiones, de esta manera se inicia con un recorrido teórico de lo que es el capital intelectual, las características de los diferentes modelos de capital intelectual, lo relacionado con sistemas de información y toma de decisiones; posteriormente se aborda la metodología empleada -para este caso fue un grupo de discusión- con el cual se logró establecer la relación entre la información pertinente al capital intelectual y su influencia en el proceso de toma de decisiones, en esta parte se explica cómo se desarrolla un grupo de discusión y se narra la experiencia particular; por último se describen y analizan los resultados obtenidos en el trabajo de campo y se cierra con un componente de conclusiones.

\section{El capital intelectual dentro de las organizaciones en la era de la información y el conocimiento}

La historia está dividida en diferentes épocas que ilustran las distintas realidades económicas, políticas y sociales generadas por algún tipo de revolución, unas más incidentes que otras. Por ejemplo, la revolución industrial marcó, a finales del siglo XVIII, una era donde los desarrollos en las maquinarias eran más importantes que la mano de obra y esto provocó un cambio en la industria que afectó fuertemente a la sociedad. Desde finales del siglo XX, debido a los grandes avances tecnológicos y a la globalización económica y comercial, se ha venido 
desarrollado una etapa en la cual predomina el conocimiento y se resalta la preponderancia del uso de la información.

Las organizaciones están inmersas en la sociedad y por ende deben adaptarse a estos grandes cambios para asumir los nuevos retos que se les presentan. Estos entes como órganos vivos en una vastedad de redes y condiciones sociales, económicas y políticas, se enfrentan a escenarios de competencia, donde al mejor estilo Darwinista, su supervivencia está atada a estructuras empresariales que además de tener buenas ideas de negocio y productos de gran demanda en el mercado, consideren desde sus entrañas la importancia de recursos que para la era industrial eran ignorados, como lo es el conocimiento y el valor de la información. A partir de estas nuevas necesidades, se emplea el término conocimiento para esbozar todo el entramado que le dio vida a lo que hoy se conoce como Capital Intelectual y se encumbra a la información, como el arma primordial con que cuentan los directivos para movilizarse y hacer frente a los requerimientos y esquemas socio-económicos de la sociedad del siglo XXI.

El capital intelectual hace su aparición en el ámbito empresarial, cuando se comienza a gestionar el recurso humano como un factor de producción clave y sensible para las organizaciones, porque en este se consignan los conocimientos, habilidades, formas de trabajo, cultura y demás características que son resaltadas en la llamada era del conocimiento y la información. Es de esta manera como se da inicio a la concepción de la importancia que tienen los intangibles que poseen las organizaciones, identificando primeramente uno de los elementos fundamentales del capital intelectual, como lo es el capital humano. Posteriormente se integra al capital intelectual el concepto de capital estructural, definido como el recurso intangible que la empresa logra hacer propio añadiéndolo a su estructura organizacional y el capital relacional que hace alusión a la relación de la empresa con los grupos de interés (Arango, Pérez y Gil, 2008).

Tras la inclusión de estos conceptos, las organizaciones que se adaptan a las dinámicas económicas han dedicado tiempo y recursos para medir y reconocer estos rubros inmateriales que poseen, porque en- 
Una aproximación a la contabilidad del capital intelectual en el proceso de toma de decisiones: un estudio en el Oriente antioqueño colombiano

tienden que en el capital intelectual se encuentra la fuente más importante de ventajas competitivas. Este componente se presenta como un elemento diferenciador con la capacidad de generar valor, como es el caso de la valoración de empresas en el mercado financiero. Como lo expresan Edvinsson y Malone:

La preponderancia del capital intelectual es inevitable, dadas las irresistibles fuerzas históricas y tecnológicas, para no hablar de los flujos de inversión, que están barriendo el mundo moderno y nos llevan hacia una economía de conocimiento. Dominará la manera como valoramos nuestras instituciones porque es el único que capta la dinámica de crear y sostener valor en las organizaciones. Es el único que reconoce que la empresa moderna cambia tan rápidamente que lo único que le queda para apoyarse es el talento y la dedicación de su gente y la calidad de las herramientas de que ésta dispone (Trad. 1998, p. 25).

\section{Metodologías de medición y reconocimiento del capital intelectual}

Establecida la importancia que tiene el capital intelectual para las organizaciones, viene a colación una tarea que hasta entonces ha revestido complejidad y para la cual se han creado diversas metodologías y se ha acudido a diferentes disciplinas; es el tema de la medición y reconocimiento del capital intelectual. Desde la última década del siglo $\mathrm{XX}$ se viene trabajando en el desarrollo de modelos que den cuenta de estos recursos no fácilmente identificables en la organización, pero es un tema de vanguardia su reconocimiento para una valoración más integral de la misma. Algunos de estos modelos se detallan en la tabla próxima, con sus aspectos más relevantes: 
Carlos Eduardo Castaño Rios, Paola Andrea Reinoso Sánchez, Jahir Alberto Zapata Gallo, John Jairo del Rio Aguirre

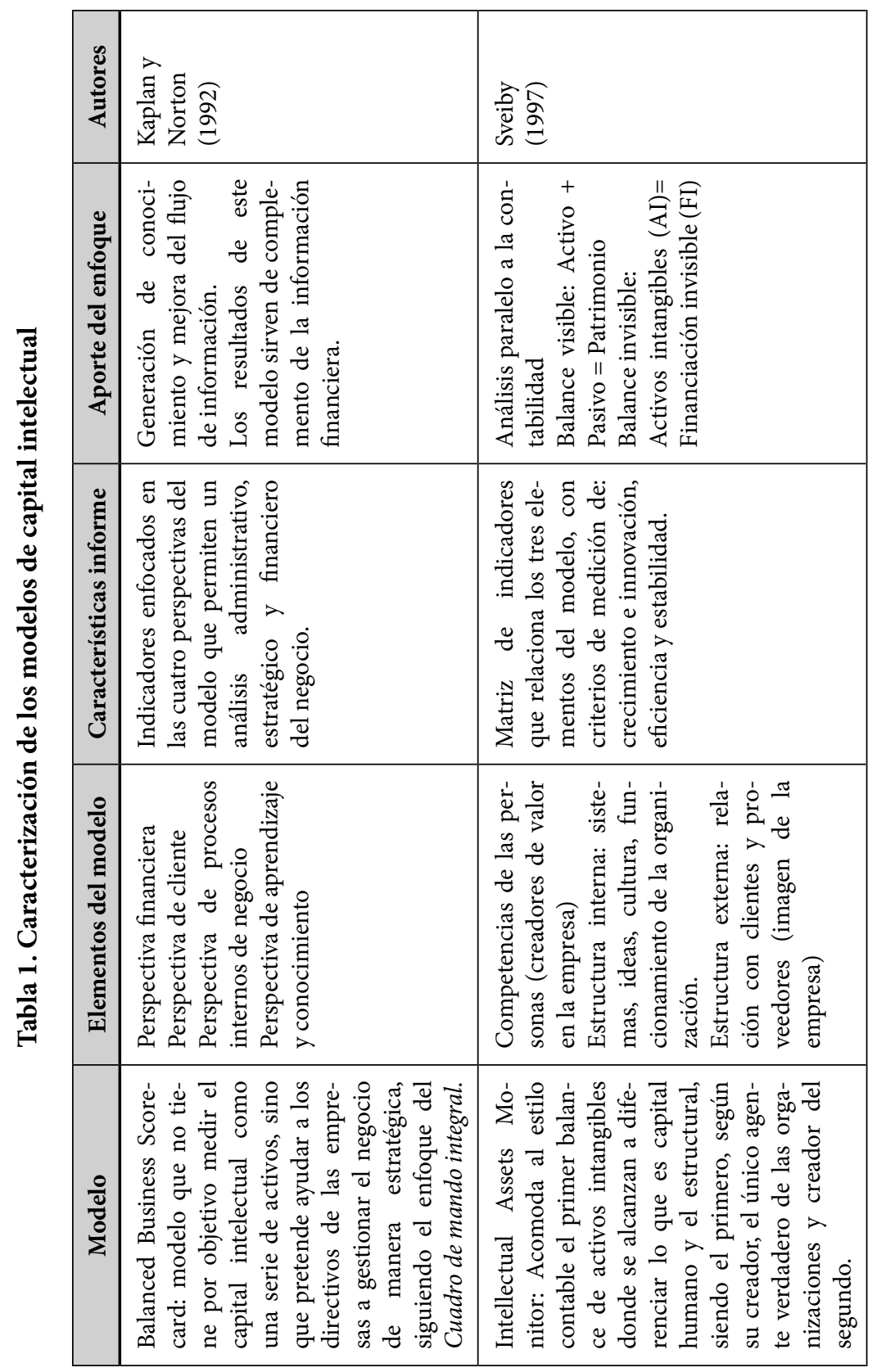


Una aproximación a la contabilidad del capital intelectual en el proceso de toma de decisiones: un estudio en el Oriente antioqueño colombiano

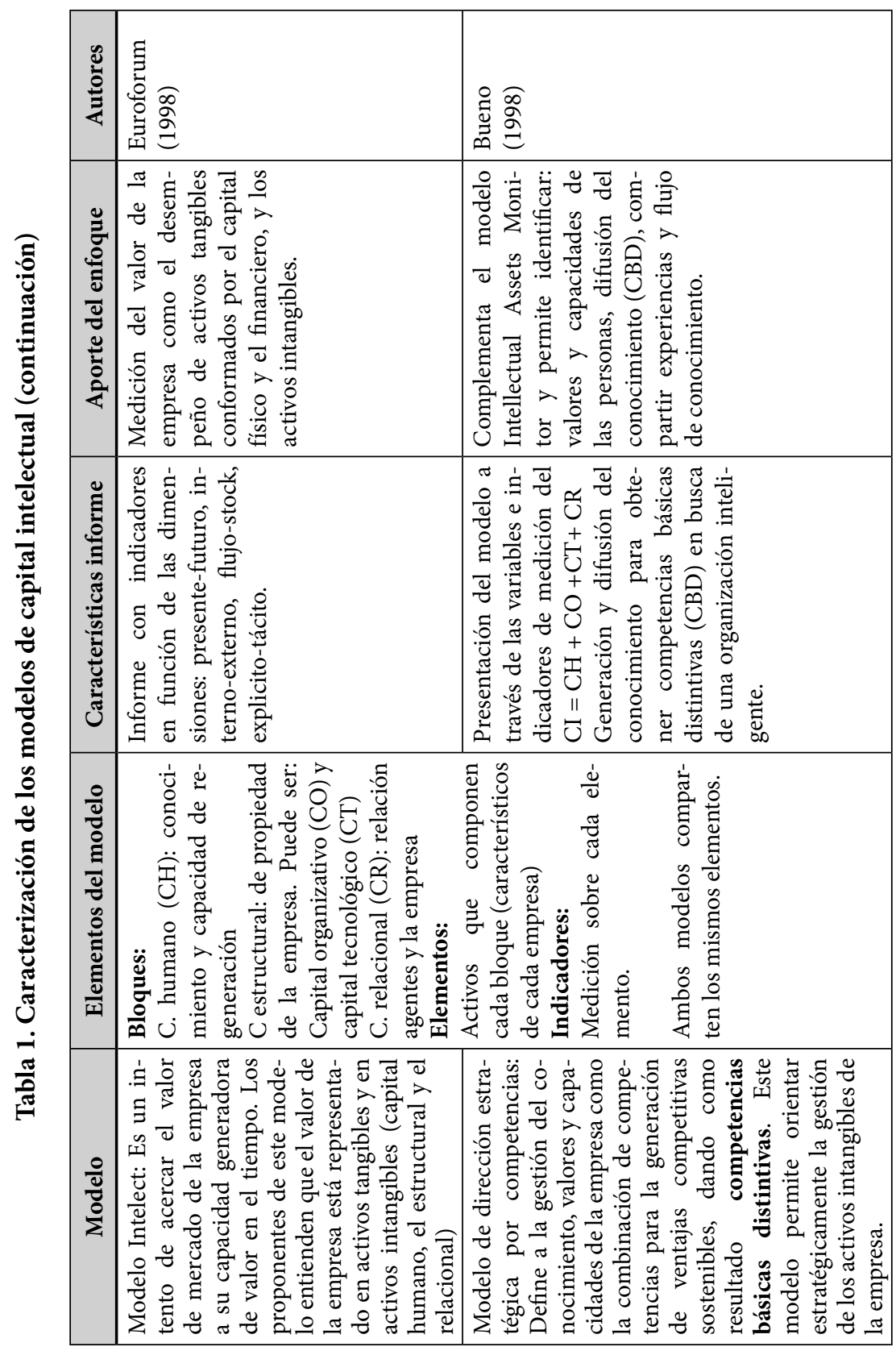


Carlos Eduardo Castaño Rios, Paola Andrea Reinoso Sánchez, Jahir Alberto Zapata Gallo, John Jairo del Rio Aguirre

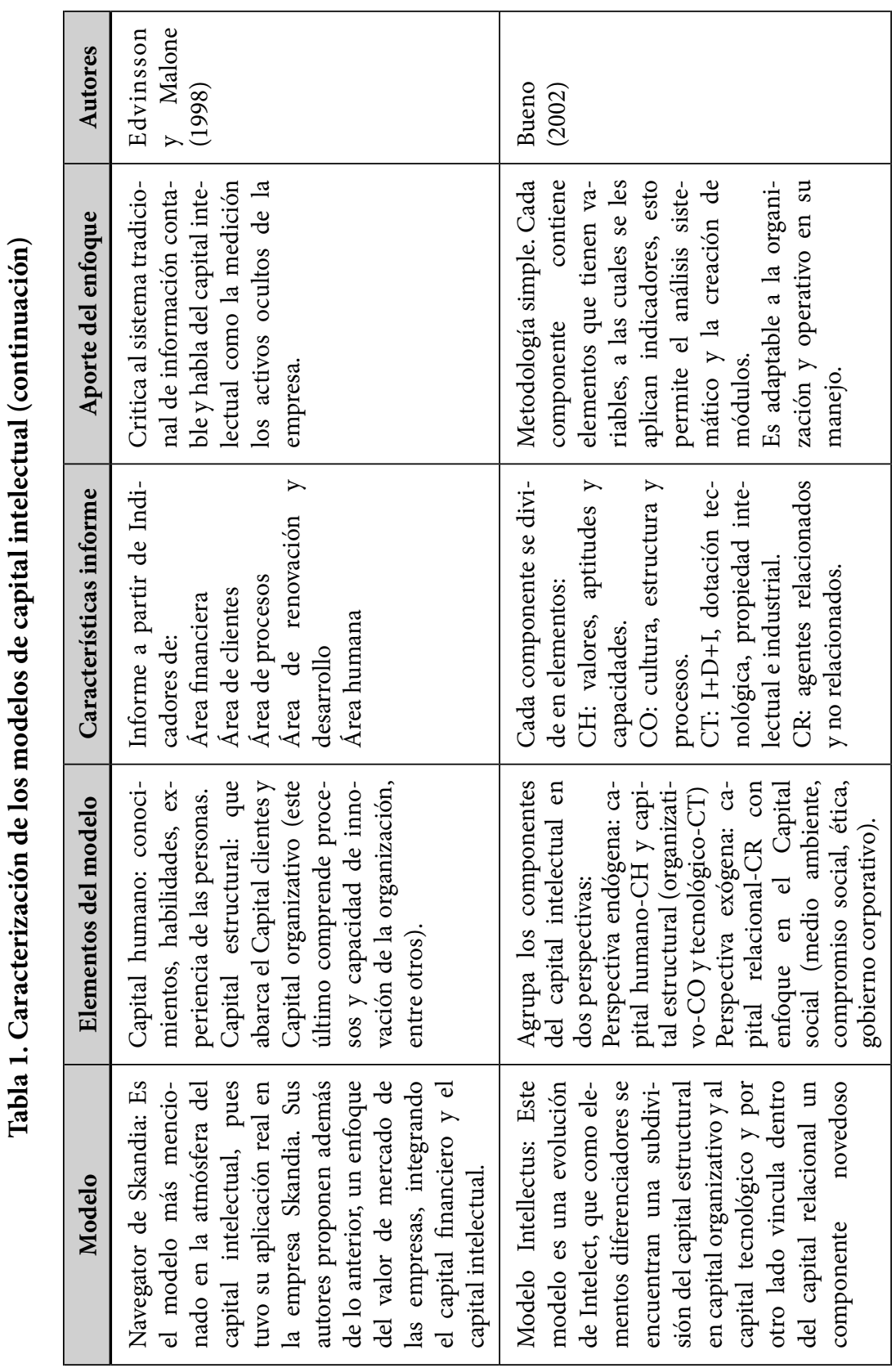


Una aproximación a la contabilidad del capital intelectual en el proceso de toma de decisiones: un estudio en el Oriente antioqueño colombiano

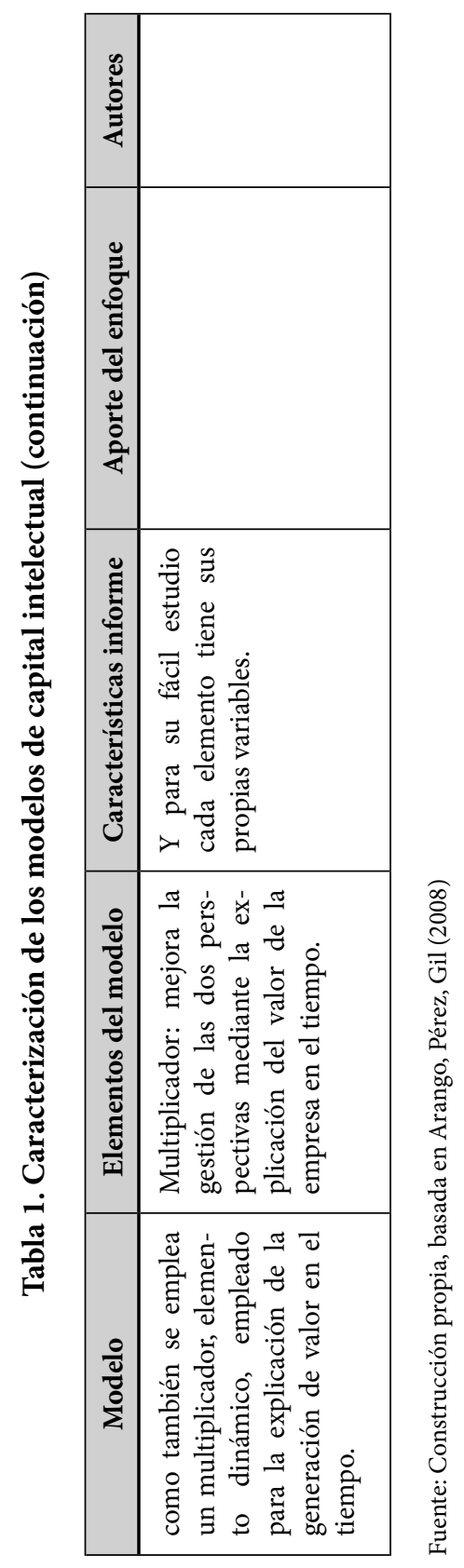


De este breve recorrido por algunas de las metodologías de medición del capital intelectual, se observa que el modelo Balanced Business Scorecard junto al modelo Navegator de Skandia atienden a perspectivas particulares por medio de indicadores enfocados al área humana, los procesos, los clientes y los impactos financieros. Esto con la diferencia de que el primero los aborda no como activos intangibles que posee la organización, sino que los consolida a través de indicadores para proporcionar un informe estratégico y administrativo, orientado a la generación de conocimiento y a la mejora del flujo de información; este informe no financiero sirve de complemento de la información financiera. El modelo Navegator por su parte, desarrolla la conceptualización del capital intelectual y divide estos activos en dos grandes grupos como lo son el capital humano y el capital estructural y, a partir de esto, busca justificar el valor de mercado de la empresa como la representación del capital financiero y el capital intelectual que ésta posee, igual a como lo supone el modelo Intelect. Estos modelos contemplan en el desarrollo del capital humano la base de creación de conocimientos, capital que medido junto a las demás competencias e infraestructura organizacional, proporcionan un mejor nivel de información para la toma de decisiones de índole estratégico y financiero.

Por su parte, el modelo Intellectual Assets Monitor se preocupa por plasmar en una estructura paralela a la contable el reconocimiento de los recursos intangibles. Este modelo amplía la gama de indicadores que permiten una valoración de la nueva estructura que propone. Posteriormente se establece el modelo de Dirección Estratégica por competencias que discrimina de manera más detallada los elementos componentes del capital intelectual y crea un mayor número de variables en sus informes que complementa el trabajo realizado en el Intellectual Assets Monitor en aras de mejorar la generación y el flujo de conocimiento al interior de la organización.

Transversal a los modelos estudiados, además del énfasis en la importancia del conocimiento y la información, se observa que la estructuración de los mismos se va articulando con más componentes o elementos a la medida que se configuran nuevos modelos y se atiende a los menesteres propios de la organización; culminando con el modelo 
Intellectus, donde se desarrolla una metodología más simple y sistémica para abarcar la mayor cantidad de variables que componen la empresa, como es el caso del capital social que no había sido explícitamente detallado en los modelos predecesores. De esta misma manera como varían las estructuras de los modelos, también lo hacen los informes resultantes de su aplicación, que a través de indicadores acerca del capital intelectual de la organización, generan información útil para que los encargados de administrar tomen decisiones.

\section{La información como un elemento esencial para la toma de de- cisiones}

La finalidad de las metodologías de medición y reconocimiento no es tarea ajena a la labor que hace la contabilidad, que como encargada de identificar, medir y revelar los recursos de las empresas, también asume la responsabilidad de informar, de forma certera y oportuna, sobre los recursos intangibles que influyen en la situación real del negocio. Aún en las primeras décadas del siglo XXI, las empresas han tenido limitantes para revelar información importante sobre los intangibles dentro la estructura de sus informes tradicionales, esto debido a las múltiples características de estos activos que dificultan la asignación de un valor específico. Como aproximación a la representación de este tipo de recursos, se podría adoptar, dentro del sistema de información contable, alguno de estos modelos que permita hacer una medición para adscribir la valoración del capital intelectual como información no financiera de tinte gerencial anexa a los informes contables tradicionales.

Como lo ilustra la figura, el sistema de información contable en términos generales es un medio que incrementa el acervo de capital estructural de la empresa, que si es adaptado según las necesidades de la organización puede cumplir de mejor manera su misión de medir los recursos e incrementar el flujo de información y conocimiento al interior de la misma, permitiendo a los altos directivos tener una visión más holística de sus compañías, para de esta manera intervenir en los factores estratégicos de sostenibilidad y competitividad a partir de una oportuna y adecuada toma de decisiones. 
Figura. Sistema de Información Contable y toma de decisiones

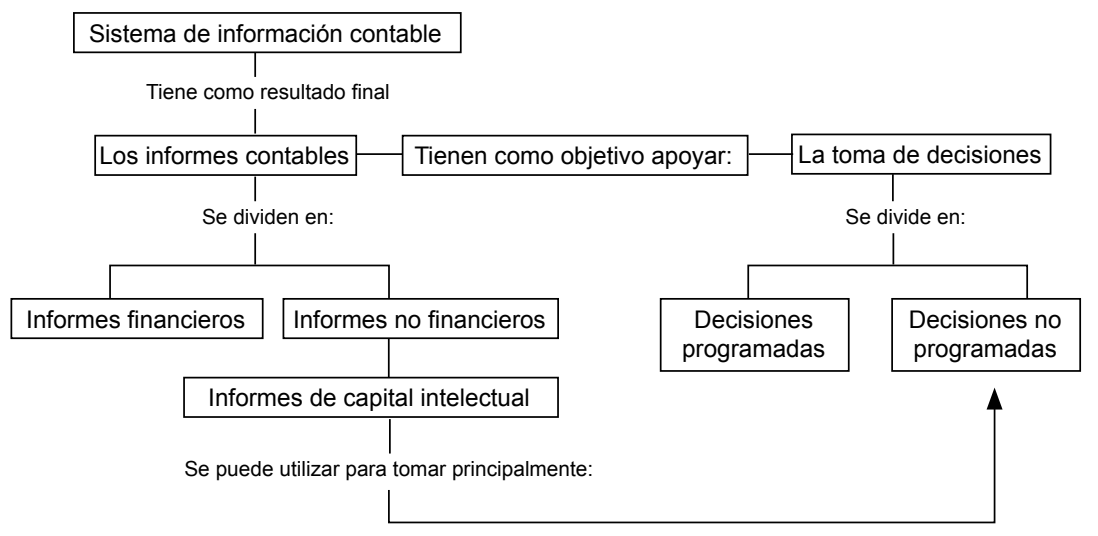

Fuente: construcción propia

Dadas las circunstancias del mercado, se debe tener un cuidado especial a la hora de gerenciar las empresas, tratando de aprovechar al máximo las fortalezas de las mismas y las oportunidades que se presenten en el entorno, buscando a la vez disminuir sus riesgos. Una manera de conseguir esta optimización se puede dar aprovechando los beneficios que brinda el sistema contable, tomando buenas decisiones, ya que estas son las que definen la dirección de una organización.

Koontz y Heinz (1998, p. 192) definen la toma de decisiones como la selección de un curso de acción entre varias alternativas, clasificando las mismas como decisiones programadas y no programadas, utilizando decisiones programadas en situaciones rutinarias y problemas estructurados, y las no programadas en situaciones no estructuradas y novedosas y enfocadas a lo estratégico. Las decisiones no programadas definen el rumbo de la empresa y aunque son decisiones muy importantes, en la mayoría de las ocasiones los gerentes o las personas encargadas de tomar este tipo de decisiones, solamente acuden a la intuición, experiencia y juicio personal para tomarlas, empero sólo en algunas organizaciones, en ciertos contextos, la toma de decisiones basadas únicamente en estos factores personales y abstractos, les puede resultar efectivo. 
Pero no siempre puede resultar efectivo tomar decisiones basándose solamente en los juicios personales dejando las decisiones a la suerte, sino que se debe tener una posición preventiva y tratar de disminuir el riesgo apoyándose en la información, tal como lo expresa Alejandro Rosado: "Las decisiones efectivas no se producen al azar y no podemos aceptar racionalmente el concepto de suerte en la decisión. La información, los conocimientos, las habilidades y la participación son factores decisivos para alcanzar la efectividad en la decisión" (1990, p. 60).

Pasar de tomar decisiones intuitivamente a tomar decisiones con base en una información más elaborada, proveniente de un sistema de información, puede ser muy beneficioso para la organización, como lo plantean Gabriel Rueda y Martha Arias: "la generación de competencias organizacionales, el manejo de incertidumbre y racionalidad, control y reacción, e innovación y adaptación, se definen como variables características de la competitividad y fundamentos de los componentes o aportes de los sistemas de información" (2009, p. 248).

\section{Aspectos Metodológicos}

La investigación realizada es de carácter cualitativo. Busca identificar las diferentes percepciones que tiene un grupo de individuos, en este caso sobre la incidencia que tiene para la toma de decisiones gerenciales el reconocimiento del capital intelectual dentro de los informes contables, para después contrastar las opiniones y retroalimentarlas.

Para cumplir con dicho objetivo se optó por desarrollar un grupo de discusión como metodología de investigación, el cual se basa según Krueger (1991, p. 24) en "una conversación cuidadosamente planeada en un ambiente permisivo no directivo, donde se cuenta con un moderador y un aproximado entre siete y diez participantes expertos en el tema, esta discusión hace que los miembros del grupo se influyan mutuamente ya que responden a lo que surge dentro de la discusión". La documentación de esta metodología condujo a la recolección de ideas que posteriormente serán tratadas a profundidad.

Este grupo de discusión se desarrolló en dos etapas partiendo la primera con una breve presentación y contextualización de los integrantes, para conocer sobre las organizaciones que representaban y 
sus funciones, además de una conceptualización de capital intelectual en la cual se entregaron elementos clave para aproximar a los asistentes del grupo hacia el tema en discusión y a continuación se realizó una segunda etapa donde se abrió la discusión a partir de una serie de preguntas abiertas donde se abordó a profundidad la incidencia de la inclusión de los informes de capital intelectual como información anexa a los informes financieros tradicionales, para que sea de utilidad para la toma de decisiones. Con la finalidad de que los datos obtenidos abarcaran la gran diversidad de la realidad empresarial y se pudieran contrastar y analizar la información suministrada por los participantes, se buscó que los asistentes fueran tomadores de decisiones de diferentes áreas organizacionales y pertenecientes a empresas de diversos sectores económicos, con la característica de que estas personas, a causa de su rol dentro de la organización, se ven enfrentados cada día con los diferentes elementos del capital intelectual. Se contó entonces, con el acompañamiento de siete profesionales con diferentes perfiles y conocimientos, pero entre los cuales coincidía que todos eran tomadores de decisiones administrativas, financieras y de recursos humanos.

La metodología desarrollada logró despertar interés sobre el tema en los participantes y generó la discusión pertinente para que se arrojaran planteamientos de interés, que funcionarán como insumo a la hora de contrastar los resultados obtenidos con el componente teórico que sustenta este trabajo.

\section{Resultados del grupo de discusión}

\section{- Etapa 1: contextualización y conceptualización}

En esta primera etapa se realizaron dos cuestionarios, el primero sobre el proceso de la toma de decisiones de los participantes en sus respectivas empresas y un segundo cuestionario con preguntas relacionadas con los elementos del capital intelectual dentro de estas entidades.

Después de aplicar el primer cuestionario a las personas participantes del grupo de discusión, se evidenció que los principales criterios tenidos en cuenta a la hora de tomar una decisión son la percepción que se tiene de la situación actual externa e interna de la empresa y la información de tipo financiero. Sin embargo no se deja de lado otro 
tipo de información que es clave para la toma de decisiones relacionada con el capital intelectual, como lo son las hojas de vida, el benchmarking y encuestas de satisfacción de clientes, que se tienen en cuenta para tomar decisiones relacionadas con el personal, la competencia y los clientes, respectivamente. Dichos tomadores de decisiones, con la información que tienen disponible, toman principalmente decisiones de financiación, impositivas, de inversión, administrativas, de personal, decisiones sobre desarrollo de nuevos productos y decisiones para expandir el negocio.

Una idea en común que se encontró, es que la información tradicional no es suficiente para la toma de decisiones en la empresa y se debe complementar con otro tipo de informes que den cuenta sobre clientes, mercado, competencia, capital humano, activos intangibles, siempre y cuando sea información que le aporte a la empresa y se pueda medir, como lo afirmó uno de los participantes: "pienso que todo recurso al ser medible, tiene posibilidades de entrar a formar parte del collage con que se cuenta para la toma de decisiones y aunque algunos no apliquen para ciertos temas, de seguro al menos le sirven mucho a su propia área para diseñar estrategias de decisión futura que finalmente afectaran todo el sistema de la compañía".

En cuanto al segundo cuestionario se observó en general que los participantes le dan gran importancia a los recursos intangibles en sus empresas, ya que los consideran totalmente necesarios y claves para el funcionamiento del negocio, sin embargo una de las personas participantes del grupo, consideró que a pesar de ser importante sólo se empieza a valorar cuando ya no se tiene, afirmando lo siguiente: "por ejemplo en el capital humano uno no aprecia sus aportes dentro de la organización hasta que la abandonan".

A pesar de que se reconoce su importancia y de que se identifican algunos recursos intangibles en las organizaciones donde los participantes toman decisiones, no existe una revelación de los mismos en algún informe, pero esto no sucede porque no sea importante sino por la complejidad que implica hacerlo o por el tipo de empresa en el que se está inmerso, como es el caso de un participante que sostiene que por el carácter público de su empresa es difícil detectarlo y por ende revelarlo.

El principal capital que identificaron y al que le dan más importancia es al capital humano, haciendo alusión al talento de los empleados 
y a la baja rotación de los mismos, ya que esto contribuye a mantener estándares de calidad altos y constantes; seguidamente identificaron el capital relacional pero delimitando el mismo hacia los clientes y por último, el capital estructural en donde se le da importancia a los software y demás sistemas de información que utilizan en sus empresas.

\section{- Etapa 2: discusión}

Los asistentes al evento observaron que sus empresas de alguna $\mathrm{u}$ otra manera están inmersas por inercia en las dinámicas económicas que se extienden al ámbito internacional, pero al traer ese modus operandi de las industrias europeas donde se aplican hoy día los informes de capital intelectual al contexto nacional, representado en las empresas invitadas, se hace evidente que el tema del capital intelectual y aún más el de los informes que dan cuenta de este recurso, es conceptualmente ignorado, aunque de forma implícita estas personas sienten que interactúan con este recurso a diario en sus actividades gerenciales.

De esta manera consideran que el capital intelectual se ha convertido en una mega tendencia presente en toda actividad económica, donde los directivos encargados de la toma de decisiones tienen por labor revaluar la tradicional forma de concebir los recursos de las empresas y por ende la función de administrar sus negocios, pues de marginar el reconocimiento del componente "capital intelectual" de sus organizaciones estarían desconociendo un elemento sensible que interviene de manera importante en la sostenibilidad y competitividad de sus empresas, y para esto se requiere de información que revele su existencia.

Los asistentes al grupo de discusión opinaron en general que aunque esta información no la utilizan actualmente como una herramienta que se emplee a la hora de administrar, pues no existe en nuestro contexto la cultura de reconocer la importancia del capital intelectual y de usarlo en pro del crecimiento organizacional, resaltan que una vez se posean conceptos claros sobre este tema y sepan de ejemplos de modelos ejecutados en otras empresas, esta información contable no financiera podrá ser útil para la toma de decisiones; pero para que esto suceda es menester que se cree cultura y la necesidad al empresario de información sobre el capital intelectual, indicándole sobre modelos versátiles traducibles a números que indiquen resultados a través de 
informes fáciles de leer y con detalle en los puntos de interés de sus actividades empresariales.

Estos informes involucran a toda la organización, por lo que aquello de crear cultura es transversal a los distintos niveles jerárquicos, donde la flexibilidad al cambio y el ambiente organizacional pueden hacer la diferencia a la hora de asimilar estas nuevas tendencias relacionadas con el capital intelectual.

\section{Los elementos del capital intelectual y la concepción de los tomadores de decisiones en las empresas}

\section{Capital humano}

Ante la concepción del capital humano en las organizaciones se evidencia que actualmente este recurso sensible para las empresas, se encuentra en una etapa donde se replantean las formas de trabajo y de valorar la actividad que realizan las personas. Tradicionalmente las empresas valoraban más a sus activos que al personal y su conocimiento, la parte humana pasaba a un segundo plano, donde el valor de este elemento radicaba en su importancia como factor de producción. Hoy día existe una tendencia de moda hacia un enfoque en las personas, tal como lo expone uno de los asistentes "gente trabajando con gente", por lo que se hace relevante saber sobre los individuos que componen la organización, conocer sobre sus características personales, sobre su entorno, sobre su perfil profesional, todo con el propósito de establecer vínculos entre los directivos y los trabajadores, y de crear un clima organizacional afable que le proporcione bienestar al humano que pone su esfuerzo en pro de la empresa. Pero esto está lejos de hacer ver a las firmas como entes altruistas frente a los trabajadores, porque en el fondo lo que hacen son pruebas en busca de un crecimiento en los índices de eficiencia y eficacia en las labores desarrolladas por estas personas, que en caso de no funcionar pasarán a ser reemplazadas por otras, convirtiendo a las organizaciones en grandes laboratorios de la conducta de los individuos frente a ciertos estímulos y situaciones ambientales. Esto se hace, como lo expresó uno de los participantes, persiguiendo la tendencia de "hacer más con menos, más con lo mismo o lo mismo con 
menos". La empresa se ocupa de las personas en lugar de preocuparse por ellas. Se invierte en los empleados en busca de beneficios económicos pues un empresario siempre lo que va a ver es dinero, efectivo y la junta directiva siempre va a estar presionando por incrementar la rentabilidad de sus negocios.

En este orden de ideas, se deja de darle tanta importancia al factor mecánico, para dirigir la mirada al elemento humano y junto a él a su conocimiento, pero como las máquinas se podían medir y controlar, ahora el aporte que hacen las personas en la empresa bajo esta perspectiva de productividad necesitan de una medición para controlar el recurso humano y poderlo administrar. Es aquí donde los participantes resaltan las bondades de tener unos informes donde se tenga en cuenta aspectos del personal de su empresa como lo son las capacitaciones, habilidades, experiencias y demás información relacionada con el capital humano; de esta manera el empresario tiene más elementos para conocer de su empresa y saber cómo tomar decisiones para dirigir sus esfuerzos y recursos financieros al crecimiento de su organización.

\section{Capital estructural}

En referencia al capital estructural se encontró que se presenta una generalización al decir que es el elemento del capital intelectual que más facilidades presenta para su reconocimiento, esto debido a las cualidades que poseen dichos recursos tales como lo son la capacidad de desarrollar productos en pro de la organización que incrementen sus ventajas tecnológicas y de información, la facilidad para ser enajenados, entre otras.

Un punto que presenta confusión en cuanto al capital estructural es la diferenciación entre lo tangible y lo intangible. La Real Academia define el concepto tangible como algo que se puede tocar y las normas técnicas contables no se salen de esta percepción; sin embargo ya que este capital puede poseer ambas formas, se vuelve complicado para los tomadores de decisiones distinguir entre éstas y más aún identificar cuándo un bien intangible puede considerarse tangible en términos de medición. Para la contabilidad un bien tangible es aquel que tiene forma física, puede ser medido y reconocido, pero las nuevas realidades 
económicas llevan a un segundo plano la primera de estas características, como se puede evidenciar en el tema capital intelectual donde cobra mayor importancia la necesidad de medir y reconocer los bienes intangibles, tengan ellos apariencia física o no.

De esta forma, lo que realmente se requiere es que se mida y reconozca el capital estructural de las empresas, para que se puedan generar informes sobre estos recursos y que el tomador de decisiones tenga dicha información en cuenta a la hora de administrar su negocio con el objetivo de que conociendo sobre la existencia de este elemento del capital intelectual, se genere un mayor beneficio a la organización, pues se hace claro para los participantes que estos recursos son lo que hoy por hoy se consideran como la mayor fuente de ventajas competitivas para las empresas. Además, son los recursos relacionados al capital estructural, los que los tomadores de decisiones dentro de sus labores cotidianas tienen más en cuenta a causa de las características de muchos de estos recursos que permiten que sean medidos y revelados en los estados financieros tradicionales, que son comúnmente empleados en las actividades gerenciales de la toma de decisiones empresariales.

\section{Capital relacional}

Como producto de la experiencia de los asistentes en el ámbito empresarial, éstos concuerdan en que son las dinámicas económicas las que marcan en el entorno el comportamiento de las organizaciones y que estos cambios influyen directamente en la toma de decisiones por parte de los encargados de esta labor, quienes ven al capital relacional con un enfoque muy dirigido hacia el cliente como razón de ser de sus negocios.

Las empresas nacen con un objeto social definido y lo que pretenden es llevar este bien o servicio hacia un consumidor, pero muchas veces es arriesgado salir a un mercado voraz sin medir la viabilidad de un proyecto empresarial, por lo anterior los asistentes exponen la necesidad de poseer información que les indique acerca de cuáles son los tipos de clientes a los que se puede llegar. Esta razón hace que este recurso revista mayor importancia para ser reflejado en algún tipo de informe y no ser manejado solo de una forma empírica. Los produc- 
tos y servicios que ofrecen las empresas siempre irán dirigidos a unas personas específicas que es necesario conocer, por ejemplo, si existe un número importante de estas personas en una zona que se pretende impactar, esta información puede existir en una base de datos y después de tenerla es necesario conocer quiénes de esas personas estarían interesadas en adquirir el producto; todo esto es capital intelectual. En estas condiciones se muestra de manera clara como bases de datos y estudios de mercados se hacen importantes y éstos pueden ser reflejados en un informe de capital intelectual que permita el análisis y facilite a los administradores la toma de decisiones.

De este modo y tras las exigencias de la época actual donde la demanda prima sobre la oferta, surge la necesidad de poseer información confiable acerca de los grupos de interés de la organización, principalmente sobre los clientes, distinguiendo estos entre potenciales, disponibles y objetivo, siendo los primeros toda la población, los segundos quienes poseen los medios y facilidades de acceder a los bienes o servicios y los últimos a quienes realmente se impactan. Los asistentes ven al capital relacional como el mayor ítem generador de beneficios dentro del capital intelectual, puesto que la relación que se posea con los clientes es la que llevará al fin a aumentar las ventas y ver de esta forma aumentada la rentabilidad, que es uno de los principales objetivos que persiguen las organizaciones.

\section{Síntesis de los resultados obtenidos}

Es evidente el cambio que han sufrido las organizaciones en las últimas décadas, cambios relacionados con su estructura jerárquica, las formas de producción, la apertura a nuevos mercados, la concepción de nuevos recursos y las estrategias que se requieren para su correcto aprovechamiento, el rol fundamental que cumple hoy por hoy el valor de la información y el conocimiento, entre otros grandes cambios que conllevan a que se acuda a nuevas formas de administrar, dando uso al sin número de herramientas que existen para la dirección organizacional. Por inercia estas entidades al verse inmersas dentro de la nueva estructura económica comienzan a sufrir reorganizaciones y a sentir la necesidad de basar sus decisiones estratégicas en nuevos elementos, 
dentro de los que se encuentran el manejo del personal, la relaciones con clientes, el vínculo entre administrativos y dueños, la generación de conocimiento, la implementación de sistemas de información y el uso de sus informes, entre otros, lo que ocasiona que de forma empírica los tomadores de decisiones tengan estos recursos como fuente primordial de su labor. Sin embargo a la hora de escuchar el término "capital intelectual" que aborda ampliamente estos elementos, se muestran sorprendidos, encontrando novedad en el tema y gran expectativa sobre el uso que le pueden dar a los informes sobre los recursos que componen el capital intelectual.

Con el ejercicio de citar a una serie de individuos con el perfil de tomadores de decisiones para que dieran sus observaciones sobre el tener en cuenta los informes de capital intelectual al momento de disponer sus esfuerzos y recursos, se evidenció que todos encuentran en estos informes una herramienta que les amplía el panorama de sus organizaciones. Los informes que observaron estas personas tienen como características, al igual que cualquier producto de muchos sistemas de información, que es construido con la colaboración de muchos eslabones que integran la organización y va dirigido a unos usuarios particulares. Estas dos características hacen que la concepción del capital intelectual tenga inferencia en todos los niveles jerárquicos para que sea apropiado por los miembros de la empresa y que sean empleados por cada segmento de la firma y, de esta manera según los criterios y menesteres de cada uno de ellos, puedan ser usados para que se tomen decisiones.

Pero aun con la posibilidad que tienen los informes de capital intelectual de atender a la diversidad de los aspectos que influyen en la organización, se observó que sin importar la función que desempeñen los tomadores de decisiones y el lugar que ocupen en las empresas, existe una tendencia a dar lectura a los elementos del capital intelectual presentes en estos informes, en el siguiente orden de importancia. Lo tercero que detallan es el capital estructural, haciendo alusión a que recursos como la imagen de la empresa, valores corporativos, las patentes y desarrollos realizados dentro de la organización, la tecnología, los canales y sistemas de información, por el hecho de que estén más explícitos en la empresa -en cuanto a su identificación se trata-, no revisten mayor novedad en su reconocimiento en los informes presen- 
tados. En segundo lugar observan al capital humano, donde resaltan recursos como lo son el conocimiento y habilidades de los empleados, capacitaciones realizadas e información sobre la administración del personal como lo es el número de trabajadores, nivel de ausentismo, rotación, entre otros; estos aspectos representaron mayor novedad e impacto para considerar dicha información aplicable al proceso de tomar decisiones. En el primer lugar de este ranking, el cual analiza la percepción que tienen los tomadores de decisiones en cuanto a la importancia de los elementos que son reconocidos en los informes del capital intelectual, estas personas ubican al capital relacional como el elemento más importante, por el hecho de revelar información sobre los clientes y porque desde la concepción de maximizar la ganancia, relacionan directamente a los clientes con las ventas y con ventas relacionan rentabilidad y son estos aspectos los que más les importan para la implementación de sus estrategias. De esta manera, consideran que los recursos del capital relacional constituyen una amalgama entre las dos tipologías de recursos antes abordados, porque el enfoque dirigido a los clientes es una tarea que involucra a toda la empresa a través del cumplimiento de objetivos organizacionales que son desarrollados por el recurso humano utilizando el componente estructural.

Sin embargo, previo a que estas personas tuvieran conocimiento acerca de los informes de capital intelectual y hubieran discutido el tema, consideraban al capital humano el elemento más significativo para sus organizaciones, puesto que conjeturan con acierto que en las personas radican los conocimientos, habilidades, valores e historia corporativa que ayudan a mantener estándares de calidad altos y que a la hora de hablar de intangibles no valorados en las empresas se evidencia con claridad que muchas veces el valor del personal es notado cuando por razones indistintas ya no está al servicio de la organización. De igual marera entienden que el capital estructural puede brindar la mayor fuente de ventajas competitivas para las empresas. Pero una vez observaron los informes de capital intelectual, constataron que estaban dejando de lado un componente cultural implícito en el acto de administrar: la concepción del beneficio económico, siendo esta una parte fundamental en la racionalidad del proceso de la toma de decisiones que está arraigado en la cultura de los individuos encargados de 
esta labor. Teniendo en cuenta esta premisa, sin importar la ocupación de estas personas y de la importancia que observen en la revelación de información referente al capital humano y estructural, volcarán su atención al capital relacional porque conciben, aunque sesgándolo solo a la parte de los clientes, que son éstos la razón de ser de sus negocios y que toda información que les permita administrar esta variable de la organización será utilizada en pro de un incremento de las ventas que se verá representado en cifras monetarias.

Tras la idea del beneficio económico, los directivos dan valor a este tipo de información que complementa la información financiera tradicional, pero además de pensar en los aspectos del capital relacional que ayudaría a elevar la rentabilidad, también encuentran otras bondades en el reconocimiento del componente humano como lo son las capacitaciones, experiencias, promoción a nuevos cargos y demás información relacionada con el capital humano que les permita ser más eficientes y eficaces en procura de una mayor productividad y el bienestar de este recurso; al igual que con el capital estructural donde se pretende identificar las fortalezas organizacionales que instauren ventajas de competencia y permitan el desarrollo de productos en pro de la organización que incrementen su acervo tecnológico y de información. De esta manera el uso de información que revelen los recursos del capital intelectual, según la perspectiva de los directivos de las empresas estudiadas, es necesaria y pertinente en el proceso de la toma de decisiones empresariales.

\section{Un salto a la era de la información, de lo empírico al papel (reco- nocimiento informado)}

El capital intelectual es un tema poco abordado por los directivos empresariales de nuestro contexto, esto a causa del escaso conocimiento que tienen sobre el tema y a que aún no sienten una expresa urgencia por tener que reconocer más elementos que les impliquen hacer cambios estructurales en sus formas de administrar, pero estas personas tampoco son ajenas a las realidades que los circundan y que les plantean exigencias para sobrevivir y no quedar anquilosados con los elementos primarios con que solían hacer frente a las distintas circunstan- 
cias de sus organizaciones y del mercado. La globalización económica, la estandarización de la información financiera, los sistemas de calidad y el libre comercio internacional, son algunos de los acontecimientos que llevan a considerar que desde lo micro a lo macro hay que estar a la vanguardia con las distintas alternativas que son ofrecidas para un mejor proceso de direccionar la organización, lo cual se puede lograr por medio de la toma de buenas decisiones, pero para que esto sea llevado a cabo se requiere de una adecuada, oportuna, fiable y útil información sobre la integralidad de la empresa. Todas estas nuevas necesidades, que anteriormente eran ignoradas, se encuentran enmarcadas en el valor que tiene la información y el conocimiento, sobre todo en una época donde se requieren de más elementos que la sola experiencia y de la información netamente financiera, para ser asertivo a la hora de tomar decisiones, que por muy pequeñas que sean pueden impactar en toda la empresa.

La articulación del capital intelectual y sus elementos como parte integral de las empresas dentro de sus sistemas de información, requiere de un estudio detallado de las diferentes metodologías de medición y reconocimiento de estos recursos ocultos en la organización; pues además de la instrucción que deben asimilar los encargados de dirigir las empresas, también deben fomentar la implementación de estas metodologías que se sintetizan en procesos de gestión de los recursos referentes a cada elemento del capital intelectual, cuyos resultados puedan ser medidos y reconocidos a través de un sistema de información que concluyan en unos informes de fácil entendimiento y aplicación, porque de nada sirve tener amplios conocimientos sobre el tema si no conducen a hacer cambios estructurales que vayan de la mano con los objetivos estratégicos de la organización. Con los informes de capital intelectual se dan más herramientas a los tomadores de decisiones acerca del universo de su empresa para que pueda construir sus estrategias directivas traducidas en decisiones que deben tomar en cada momento de su diario acontecer.

Una de las opciones que se tiene a la hora de reconocer los elementos del capital intelectual es a través de informes basados en variables que se diseñan según la información requerida por la empresa. Estos informes son el producto de la aplicación de algún modelo de capital 
intelectual. La diversidad de la información que se recolecta con estos modelos sirve para atender a las particulares decisiones que se deben tomar a diario por parte de los diferentes segmentos de la organización. De esta manera la flexibilidad de adaptar los distintos modelos de medición y reconocimiento del capital intelectual a las necesidades de las empresas, permite que se obtenga información particular para proporcionar soluciones específicas a través de la toma de decisiones puntuales.

Los sistemas de información de las empresas son el insumo por excelencia para los directivos de las organizaciones a la hora de conocer la radiografía de sus negocios y dentro de estos se encuentra principalmente la contabilidad financiera que, a través de sus informes financeiros, busca reflejar la situación actual de la organización, pero se observa que estas personas frecuentan otro tipo de información de índole estadístico, cualitativo y referente al corazón de sus entidades que no aborda rubros monetarios y que permite ampliar su percepción sobre el presente y porvenir de sus empresas para con base en estos datos ejecutar sus formas de dirección. En este sentido, la medición y reconocimiento del capital intelectual se presenta como alternativa de revelar el valor oculto que existe en las organizaciones, valor que se escapa de la información histórica del reconocimiento contable, pero que se proyecta en el tiempo como el recurso más importante que tiene gran aplicabilidad al contexto de la organización, además, como lo expresa Edwinson y Malone: "si bien la contabilidad tradicional se hizo adrede como un instrumento de presentación del pasado, el capital intelectual es un instrumento de navegación por el futuro" (Trad. 1998, p. 75) y añade más adelante: "En lugar de reemplazar el sistema actual de medidas financieras, que es producto de muchas generaciones, la medición de capital intelectual lo complementa y lo aumenta" (Trad. 1998, p. 234). De esta manera, el papel conjunto que juega el capital intelectual y la contabilidad, permite que la información financiera tradicional sea complementada con los informes de capital intelectual para que los juicios de los usuarios de dicha información sean mejor informados y ajustados a la realidad empresarial (razonables).

También es de observar que esos juicios o decisiones que son tomados en las distintas actividades de las empresas son una escogencia 
entre múltiples caminos y que se pueden realizar atendiendo a hechos programados o no en la organización. Para los primeros, suelen ser empleados los procesos estructurados que se poseen, así como también la experiencia de los directivos. Con los hechos no programados que pueden definir el rumbo de la empresa, los encargados de seleccionar el camino a seguir tienden a acudir a cuanta información tengan a la mano, pero primordialmente hacen caso a su intuición, donde de manera implícita son tenidos en cuenta los conocimientos y recursos intangibles con que cuenta la organización. La combinación de experiencia, intuición e información en los eventos no convencionales puede representar el éxito o el fracaso en el proceso de decisión, pero es sabido que cuanto mayor sea la información de calidad que se posea, mayores las posibilidades elegir con acierto. Dichos tomadores de decisiones, tal como se mencionó anteriormente, deciden principalmente sobre asuntos de financiación, impuestos, inversión, disposiciones administrativas, de personal, sobre los clientes, entre otros. Aunque la principal decisión que deben tomar estos individuos, para efectos del presente trabajo y dado el caso, es sobre la pertinencia de incluir o no la concepción, metodologías y procesos de información que ofrece el capital intelectual con el propósito de que se vea plasmado en informes contables, eso que manejan intuitivamente los tomadores de decisiones.

Ante los rápidos cambios que tienen que enfrenar las organizaciones, no es de juzgar que los directivos consideren al capital intelectual como un tema pasajero o una moda del momento, al catalogarlo como una mega tendencia. Lo cierto es que en los contextos europeos ya es una necesidad saber sobre este tipo de información y una exigencia para las grandes corporaciones. Además, no se trata de un tema nuevo, puesto que de entrada ya está inmerso en la intuición del administrador y lo que se busca con revelarlo a través de informes de capital intelectual; es pasar de lo empírico al papel, es estructurar y dar vida a una riqueza inmaterial que existe en medio de los procesos, sistemas y canales de información, clima organizacional, talento individual y colectivo, tecnología, entre otras características que posee la empresa en pro de la sinergia de los esfuerzos donde interaccionan las personas y los elementos internos y externos de la organización. Convertir todo este conocimiento en información es lo que pretende la contabilidad del ca- 
pital intelectual y por esta razón existe gran diversidad de metodologías y de teóricos, como lo son Edvinsson y Malone (1998), Bueno, Salmador y Merino (2008) y Cañibano (2012), quienes están convencidos, sin necesidad de ser catalogados como futuristas, de que la información sobre el capital intelectual acerca a las empresas a su verdadero valor, que se puede potenciar una vez descubierto y con el que se puede lograr éxito en la lid de la organización por permanecer y ser cada día más competitiva en su entorno. Por este motivo la contabilidad como sistema de información que refleja la realidad organizacional debe generar sinergias con el capital intelectual, para que articulando a sus procesos las metodologías de medición y reconocimiento de este valioso recurso, pueda estar a la vanguardia y satisfacer a los tomadores de decisiones de las necesidades de información de aquellos intangibles que representan valor para la empresa.

Entonces, ¿por qué los informes de capital intelectual no son aplicados en nuestro contexto? La respuesta a este interrogante radica en la cultura de los tomadores de decisiones, puesto que, al no concebir a los informes de capital intelectual como un insumo valioso y trascendental para sus procesos de dirección a causa de su desconocimiento, tampoco lo considerarán una necesidad. Por lo que es menester que estas personas estén al tanto de la importancia que estos recursos tienen para la organización. Pues cuando estos individuos tienen en sus manos una muestra de estos informes expresan que son pertinentes y de gran incidencia en la forma de administrar y resaltan que no es lo mismo tomar decisiones sobre la información que poseen por la sola intuición producto de su experiencia a tomarlas con una información específica y estructurada de una manera especial que permita una toma de decisiones más racional y puntual.

\section{Conclusiones}

Las organizaciones como entes inmersos en una sociedad cambiante se han visto obligadas a evolucionar a la par de las dinámicas socio-económicas, de igual forma las decisiones que toman los administradores de estas empresas deben adaptarse a la estructura que el medio les exige con el objetivo de cumplir con una de sus principales finalidades, 
la cual es permanecer en el tiempo. Es de notar que desde finales del siglo XX la sociedad atraviesa la denominada era del conocimiento y la información en donde el tema del capital intelectual se presenta como una fuente de recursos muy importante, los cuales son grandes generadores de ventajas competitivas y de valor en las empresas, por lo que los directivos deben tenerlos en cuenta a la hora de adoptar sus estrategias $\mathrm{y}$ tomar sus decisiones.

Las decisiones no programadas son las que definen las estrategias organizacionales y, por ende, las empresas deben contar con la mayor cantidad de información de forma oportuna y de calidad. En este sentido la contabilidad como principal fuente de información en las organizaciones se ve en la necesidad de hacerse cargo de presentar información que dé cuenta de la integralidad de la empresa donde se revelen los recursos intangibles que influyen en la situación real del negocio y por tanto presentan gran incidencia en el proceso de tomar decisiones.

La contabilidad como sistema de información que refleja la realidad organizacional debe articular al capital intelectual a sus procesos, aprovechando la flexibilidad que tienen las diferentes metodologías de medición y reconocimiento de este valioso recurso para ser adaptadas a las necesidades de las empresas. De esta manera, se incrementa el poder informativo de la contabilidad al proporcionar informes de capital intelectual como información contable no financiera que permita satisfacer las demandas de información de los individuos encargados de disponer de los esfuerzos y recursos organizacionales.

El reconocimiento del capital intelectual dentro de los informes contables tiene como principal implicación para la toma de decisiones que proporciona una visión más amplia del panorama de los recursos presentes en la organización al ser reconocidos a través del capital relacional, el capital humano y el capital estructural. La información que se genera alrededor del capital intelectual está al servicio de la organización, lo cual permite una toma de decisiones más completa, racional y puntual.

Este trabajo de investigación se permitió evidenciar que los tomadores de decisiones tienen en cuenta información de tipo no financiero sobre sus clientes, empleados y procesos empresariales, lo que permite aseverar que estas personas consideran de manera implícita dentro de 
su labor los recursos del capital intelectual, aunque ignoran la existencia del término y de los ítems que lo componen. Pese a esto, después de los directivos tener un acercamiento e información pertinente al tema, consideran al capital intelectual y sus informes realmente importantes y piensan que podría cambiar de manera positiva la forma de tomar decisiones. Además de lograr esto, afirman que estos informes podrían tener impacto en la organización en cuanto a la relación con los diferentes grupos: se aumentaría el compromiso de los directivos, se elevaría el sentido de pertenencia por parte de los empleados y se lograría fortalecer las relaciones y la satisfacción con los clientes.

Por último, gracias a la característica que tiene la información de capital intelectual de ser complementaria a la información financiera, se puede aprovechar de gran manera por parte de la los directivos para contrastar estos dos tipos de información por medio de estudios de gran interés para la organización, como lo puede ser analizar si el incremento de la utilidad está ocasionado por el aumento en la satisfacción de los empleados y/o clientes, contemplar la posibilidad de nueva contratación de personal y la promoción del mismo y programar las capacitaciones de los empleados, entre otros. Son grandes los beneficios que podrá generar esta información contable no financiera a las organizaciones, pero para que esto suceda es menester que en el entorno empresarial actual se cree la cultura y la necesidad al empresario de utilizar información relacionada con el capital intelectual a la hora de gerenciar sus organizaciones.

\section{Referencias}

ÁLVAREZ, C.P \& Piedrahita, R.A (2002). El capital intelectual como base de ventajas competitivas sostenibles: gestión, medición, y contabilización del capital intelectual en la compañía CONHYDRA S.A. E.S.P. Tesis de especialización para optar al Título de Revisor fiscal, Facultad de Ciencias Económicas, Universidad de Antioquia, Medellín, Colombia.

ARANGO, M. D., Pérez, G. \& Gil, H. (2008). Propuestas de modelos de gestión de capital intelectual: Una revisión. Contaduría Universidad de Antioquia, 52, 105-130. 
BUENO, E., Salmador, M. \& Merino, C. (2008). Génesis, concepto y desarrollo del capital intelectual en la economía del conocimiento: Una reflexión sobre el Modelo Intellectus y sus aplicaciones. Estudios de Economía Aplicada, Agosto, 43-63. Extraído el 12 de Noviembre del 2012 de: http://redalyc. uaemex.mx/redalyc/src/inicio/ArtPdfRed.jsp?iCve=30113187003\#

CAÑIBANO, L. (2012). La relevancia de los intangibles en la información financiera. Contaduría Universidad de Antioquia, 60, 40-54.

CIC. (2002). Guías y Directrices de Utilización del Modelo Intellectus. Documento Intellectus, Centro de Investigación sobre la Sociedad del Conocimiento. Universidad Autónoma de Madrid.

EDVINSSON L \& Malone M. (trad. 1998) El capital intelectual como identificar y calcular el valor inexplorado de los recursos intangibles de su empresa. Bogotá: Norma.

EUROFORUM Escorial. (1998). Medición del Capital Intelectual. Modelo Intelect. Madrid: Ed. I.U.

FLORES, G. (2001). Contabilidad financiera: capital intelectual en el ámbito de la contabilidad financiera: revisión del caso Chileno. Revista legis del contador, p. 79-116.

GALLARDO, D. (2001). Capital intelectual y gestión del conocimiento: Problemática contable. Partida doble, 127, 58-71.

GARCÍA, A. (2007). El capital intelectual y su impacto en las organizaciones Mexicanas. Universidad de Illes Balears.

HILL, C. \& Jones, G. (2009). Liderazgo estratégico. En McGraw-Hill Interamericana Editores S.A de C. V (Ed), Administración estratégica (pp 1-33). México D.F, México.

KAPLAN, R. \& Norton, D. (1992). The Balance Scorecard: Measures that Drive Performance. Harvard Business Review, 71-79.

KNOWLEDGE. Berrett-Koehler Publishers Inc. Traducido al español por Mazars (2000), bajo el título: La nueva riqueza de las empresas. Cómo medir y gestionar los activos intangibles para crear valor. París: Gestión 2000.

KOONTZ, H. \& Weihrich, H. (1998), Administración una perspectiva global (8 va ed.) México: McGraw-Hill.

KRUEGER, Richard, El grupo de discusión. Guía práctica para la investigación aplicada Madrid, Pirámide (s.f.) 
Una aproximación a la contabilidad del capital intelectual en el proceso de toma de decisiones: un estudio en el Oriente antioqueño colombiano

NASTASI, A. (2006). El informe contable. Boletín de lecturas sociales y económicas UCA N 26. Argentina, 55-59.

NEVADO, D. \& López, V. (2000); ¿Cómo medir el capital intelectual de una empresa? Partida doble, 115, 42-53.

RAMÍREZ, D. E. (2007). Capital intelectual. Algunas reflexiones sobre su importancia en las organizaciones. Pensamiento \& Gestión, 131-152.

RIVERO, D. y Gutiérrez A. (2008). Capital intelectual. Origen, evolución y desarrollo. Conceptualización, Otros conceptos de economía. Extraído el 12 de Noviembre, 2012 de: http://www.gestiopolis.com/economia/capital-intelectual.htm

SIERRA, G. J \& Moreno, I. (2000). La información contable sobre el capital humano. Partida doble, 116, 60-71.

UNIVERSIDAD AUTÓNOMA DE MADRID (2000). Entrevista y grupo de discusión. Extraído el 2 de Noviembre del 2012 de:

http://www.uam.es/personal_pdi/stmaria/jmurillo/Met_Inves_Avan/Presentaciones/Entrevista_Guposdiscusion.pdf

FERRELL O.C., Hirt, G., Ramos, L., Adriaenséns. M. \& Flores, M. A. (2004). Introducción a los Negocios en un Mundo Cambiante. McGraw-Hill.

ROSADO, A. (1990). El proceso de toma de decisiones como instrumento de ayuda al supervisor. Salud uninorte, 6-7 (2) ,57-64.

RUEDA, G. \& Arias, M, L. (2009). Los sistemas de información contable en la administración estratégica organizacional. Cuadernos de contabilidad, 10 (27), 247-268. 